\title{
FIELDS OF CONSTANTS OF INFINITE HIGHER DERIVATIONS
}

\author{
JAMES K. DEVENEY 1
}

\begin{abstract}
Let $K$ be a field of characteristic $p \neq 0$, and let $P$ be its maximal perfect subfield. Let $h$ be a subfield of $K$ containing $P$ such that $K$ is separable over $h$. We prove: Every regular subfield of $K$ containing $h$ is the field of constants of a set of higher derivations on $K$ if and only if (1) the transcendence degree of $K$ over $h$ is finite, and (2) $K$ has a separating transcendency basis over $h$. This result leads to a generalization of the Galois theory developed in [4].
\end{abstract}

I. Introduction. Let $K$ be a field of characteristic $p \neq 0$, and let $P$ be its maximal perfect subfield. If $h$ is the field of constants of a set of higher derivations on $K$, then $h$ is a regular subfield of $K$ containing $P$. This paper is concerned with determining when every regular subfield of $K$ containing $h$ (and hence $P$ ) is the field of constants of a set of higher derivations on $K$. Necessary and sufficient conditions are shown to be (1) the transcendence degree of $K$ over $h$ is finite, and (2) $K$ has a separating transcendency basis over $h$. This is Corollary (4.2). This result leads to an immediate extension of the Galois theory developed in [4]. In part, we can restate the main result of [4] as follows: Assume $K$ has a finite separating transcendency basis over a subfield $h$ containing $P$. Then there exists a one-to-one correspondence between regular subfields of $K$ containing $h$ and Galois subgroups of $H_{h}^{\infty}(K)$. (The characterization of Galois subgroups remains the same.) Moreover, (4.2) shows this to be the most general condition on $K$ relative to $h$ under which all regular subfields of $K$ containing $h$ will be fields of constants of groups of higher derivations on $K$.

II. Definitions and preliminary results. Throughout this paper, $K$ will be a field of characteristic $p \neq 0$. A higher derivation on $K$ is a sequence $d=\left\{d_{i} \mid 0 \leqq i<\infty\right\}$ of additive maps of $K$ into $K$ such that

$$
d_{r}(a b)=\sum\left\{d_{i}(a) d_{j}(b) \mid i+j=r\right\}
$$

and $d_{0}$ is the identity map. The set $H^{\infty}(K)$ of all higher derivations on $K$

Received by the editors March 15, 1973.

AMS (MOS) subject classifications (1970). Primary 12F99.

Key words and phrases. Higher derivation, regular extension field.

${ }^{1}$ This work was supported in part by a grant from the Florida State University Committee on Faculty Research Support under the direction of Dr. Nicholas Heerema. 
is a group with respect to the composition $d \circ e=f$ where

$$
f_{i}=\sum\left\{d_{m} e_{n} \mid m+n=i\right\}
$$

[1, Theorem 1, p. 33]. Note that the first nonzero map (of subscript $>0$ ) is a derivation. The field of constants of a subset $G \subseteq H^{\infty}(K)$ is $\left\{a \in K \mid d_{i}(a)=\right.$ $\left.0, i>0,\left(d_{i}\right) \in G\right\} . H_{h}^{\infty}(K)$ will denote the group of all higher derivations on $K$ whose field of constants contains the subfield $h$.

(2.1) [2, Theorem 1]. Let $B$ be a $p$-basis for $K$ and let $f: A \times B \rightarrow K$ be an arbitrary function. There is a unique $\left(d_{i}\right) \in H^{\infty}(K)$ such that for each $b \in B$ and $i \in Z, d_{i}(b)=f(i, b)$.

(2.2) [3, p. 436]. Let $\left(d_{i}\right) \in H^{\infty}(K)$ and $a \in K$. Then $d_{i p}\left(a^{p}\right)=\left(d_{i}(a)\right)^{p}$ and if $p$ and $j$ are relatively prime, $d_{j}\left(a^{p}\right)=0$.

A field $K$ is a regular extension of a subfield $h$ if $K / h$ is separable and $h$ is algebraically closed in $K$.

(2.3) [4, Theorem 2.3]. Let $h$ be the field of constants of a set of higher derivations on $K$. Then $K$ is a regular extension of $h$.

(2.4) Lemma. The field of constants of $H^{\infty}(K)$ is $P$, the maximal perfect subfield of $K$.

Proof. Let $\alpha \in K \mid P$. If $\left\{\alpha, \alpha^{p^{-1}}, \alpha^{p^{-2}}, \cdots\right\} \subseteq K$, then $P\left(\alpha, \alpha^{p^{-1}}, \alpha^{p^{-2}}, \cdots\right)$ would also be perfect, contrary to the assumption that $P$ is maximal. Thus there exists $n \geqq 0$ such that $\alpha^{p^{-n}} \in K \backslash K^{p}$. Let $\left\{\alpha^{p-n}\right\} \cup T$ be a $p$-basis for $K$, and define $d=\left\{d_{i}\right\}$ by $d_{1}\left(\alpha^{p^{-n}}\right)=1, d_{1}(t)=0 \forall t \in T, d_{i}(x)=0$, $x \in\left\{\alpha^{p^{-n}}\right\} \cup T, 1<i<\infty$. Then

$$
d_{p^{n}}(\alpha)=d_{p^{n}}\left(\left(\alpha^{p^{-n}}\right)^{p^{n}}\right)=\left(d_{1}\left(\alpha^{p^{-n}}\right)\right)^{p^{n}}=1
$$

by (2.2). Thus the field of constants of $H^{\infty}(K)$ is contained in $P$. Applying (2.2) shows $P$ is contained in the field of constants of $H^{\infty}(K)$, and the lemma is established.

III. Higher derivations and separating transcendency bases. As before, $K$ is a field of characteristic $p \neq 0$ with maximal perfect subfield $P$. Throughout this section we assume the transcendence degree of $K / P(\operatorname{tr} \mathrm{d}(K / P))$ is finite.

(3.1) THEOREM. Let $K \supseteq h \supseteq P$ be fields and assume $K$ has a separating transcendency basis over $h$ and $h$ is algebraically closed in $K$. Then $h$ is the field of constants of a set of higher derivations on $K$.

Proof. Let $\left\{x_{1}, \cdots, x_{n}\right\}$ be a separating transcendency basis, and hence a relative $p$-basis [5, Theorem 15, p. 384], for $K / h$, and let $T$ be a $p$-basis for $h$. Since $K / h$ is separable, $\left\{x_{1}, \cdots, x_{n}\right\} \cup T$ is a $p$-basis for $K$. Define $\mathscr{F}=\left\{d^{1}, \cdots, d^{n}\right\}$ by $d_{1}^{i}\left(x_{i}\right)=1, \quad d_{1}^{i}(x)=0, \quad x \in\left\{x_{1}, \cdots, \hat{x}_{i}, \cdots, x_{n}\right\} \cup T, 1 \leqq i \leqq n$, 
and

$$
d_{j}^{i}(x)=0, \quad x \in\left\{x_{1}, \cdots, x_{n}\right\} \cup T, 1 \leqq i \leqq n, 1<j<\infty .
$$

Since $d_{j}^{i}(t)=0 \forall t \in T, 1 \leqq i \leqq n, 1 \leqq j \leqq \infty, h$ is contained in the field of constants of $\mathscr{F}$. By [4, Theorem 3.2], the transcendence degree of $K$ over the field of constants of $\mathscr{F}$ is $n$, and hence $h$ is the field of constants of $\mathscr{F}$.

(3.2) Example. The condition of Theorem (3.1) is not necessary. Consider the following example [5, Example 10, p. 389]. Let $P$ be a perfect field and $Z=\left\{z_{1}, z_{2}, \cdots\right\}$ a denumerable set of elements algebraically independent over $P$. Let $P\left(Z^{p-\infty}\right)$ be the perfect field

$$
P\left(Z^{p-\infty}\right)=P\left(Z, Z^{p^{-1}}, Z^{p^{-2}}, \cdots\right) .
$$

Let $y, u_{0}$ be algebraically independent over $P\left(Z^{p-\infty}\right)$, and define quantities $u_{n}$ recursively by

$$
u_{n}=y^{\nu^{n-1}}+Z_{n} u_{n-1} \quad(n=1,2, \cdots) .
$$

Let $K=P\left(Z^{p-\infty}, y, u_{0}, u_{1}^{p^{-1}}, u_{2}^{p-2}, \cdots, u_{n}^{p-n}, \cdots\right)$.

Mac Lane has shown $P\left(Z^{p-\infty}\right)$ is the maximal perfect subfield of $K$, $K$ has $\left\{y, u_{0}\right\}$ as a transcendency basis over $P\left(Z^{p-\infty}\right)$, and $\{y\}$ is a $p$-basis for $K$. Thus $K$ does not have a separating transcendency basis over $P\left(Z^{p-\infty}\right)$, but by (2.4), $P\left(Z^{p-\infty}\right)$ is the field of constants of $H^{\infty}(K)$.

This example also shows that not every regular subfield $h$ of $K$ containing $P$ is the field of constants of a set of higher derivations. Let $h$ be the algebraic closure of $P\left(Z^{p-\infty}, y\right)$ in $K$. Since $\{y\}$ is $p$-independent in $K, K / h$ is separable and hence regular. Since $\operatorname{tr} \mathrm{d}\left(K / P\left(Z^{p-\infty}\right)\right)=2, h \neq K$. Since $\{y\}$ is a $p$-basis for $K$, the null set $\varnothing$ is a relative $p$-basis for $K / h$ and hence by $(2.1) H_{h}^{\infty}(K)=\{0\}$ and $h$ is not the field of constants of any set of higher derivations on $K$.

Let $h$ be a subfield of $k$ containing $P$ such that $K$ is separable over $h$ and assume $\operatorname{tr} \mathrm{d}(K / h)<\infty$.

(3.3) THEOREM. Every regular subfield $k$ of $K$ containing $h$ is the field of constants of a set of higher derivations on $K$ if and only if $K$ has a separating transcendency basis over $h$.

Proof. If $K$ has a separating transcendency basis over $h$, then $K$ has one over any regular subfield $k$ containing $h$ [5, Theorem 18, p. 387], and hence every regular subfield $k$ containing $h$ is the field of constants of a set of higher derivations (3.1). Conversely, assume $K$ does not have a separating transcendency basis over $h$. Let $T$ be any relative $p$-basis for $K$ over $h$. Since $T$ is algebraically independent over $h$, in view of [5, Theorem 13, p. 383], $T$ cannot be a transcendency basis for $K$ over $h$. 
Thus if we let $k$ be the algebraic closure of $h(T)$ in $K, k \neq K, k$ is a regular subfield of $K$ (since $K / k$ preserves $p$-independence) and as in (3.2) $H_{k}^{\infty}(K)=$ $\{0\}$. Thus the theorem follows.

(3.4) COROLlary. The following are equivalent.

(1) There exists a transcendency basis $T$ for $K$ over $h$ such that $K^{p^{n}}$ is a separable extension of $h(T)$.

(2) Every regular subfield of $K$ containing $h$ is the field of constants of a set of higher derivations on $K$.

(3) K has a separating transcendency basis over $h$.

Proof. The equivalence of (1) and (3) is [5, Theorem 6, p. 375]. (3.3) shows (2) equivalent to (3).

IV. Transcendence degree of $K / h=\infty$.

(4.1) Theorem. Let $K$ be a field of characteristic $p \neq 0$. Let $h$ be $a$ subfield of $K$ containing $P$ such that $K$ is separable over $h$ and assume the transcendence degree of $K$ over $h$ is infinite. Then there exists a regular subfield $k$ of $K$ containing. $h$ which is not the field of constants of any set of higher derivations on $K$.

Proof. Let $T$ be any relative $p$-basis for $K$ over $h$. If $|T|<\infty$, let $k$ be the algebraic closure of $P(T)$ in $K$. Then $K$ is regular over $k(K / k$ preserves $p$-independence) and since $\varnothing$ is a relative $p$-basis for $K$ over $k$, $H_{k}^{\infty}(K)=\{0\}$ and $k$ is the desired subfield. If $|T|=\infty$, let $T=\left\{x_{1}, x_{2}, \cdots\right\} \cup S$. Let $k_{1}$ be the algebraic closure of $h(s)$ in $K$. Then $\left\{x_{1}, x_{2}, \cdots\right\}$ is a relative $p$-base for $K$ over $k_{1}$. Elementary calculations show $\left\{x_{1} x_{2}^{p}, x_{2} x_{3}^{p}, \cdots\right.$, $\left.x_{n} x_{n+1}^{p}, \cdots\right\}$ is also a relative $p$-basis. Since $K / k_{1}$ is separable, $\left\{x_{1} x_{2}^{p}\right.$, $\left.x_{2} x_{3}^{p}, \cdots\right\}$ is algebraically independent over $k_{1}$. We claim $\left\{x_{1}, x_{1} x_{2}^{p}\right.$, $\left.x_{2} x_{3}^{p}, \cdots\right\}$ is also algebraically independent over $k_{1}$. If not, $\left\{x, x_{1} x_{2}^{p}, \cdots\right.$, $\left.x_{n-1} x_{n}^{p}\right\}$ must be algebraically dependent over $k_{1}$ for some $n$. But $k_{1}\left(x_{1}, x_{2}, \cdots, x_{n}\right)$ is algebraic over $k_{1}\left(x_{1}, x_{1} x_{2}^{p}, \cdots, x_{n-1} x_{n}^{p}\right)$ and hence

$$
\operatorname{tr} \mathrm{d}\left(k_{1}\left(x_{1}, \ldots, x_{n}\right) / k_{1}\right)<n,
$$

a contradiction. Thus $\left\{x_{1}, x_{1} x_{2}^{p}, \cdots\right\}$ is algebraically independent over $k_{1}$. Let $k$ be the algebraic closure of $k_{1}\left(x_{1} x_{2}^{p}, x_{2} x_{3}^{p}, \cdots\right)$ in $K$. Then $k$ is a regular subfield of $K, k \neq K$, and $\varnothing$ is a relative $p$-basis for $K$ over $k$. Thus $H_{k}^{\infty}(K)=\{0\}$ and $k$ is not the field of constants of any set of higher derivations on $K$.

(4.2) Corollary. Let $K$ be a field of characteristic $p \neq 0$. Let $h$ be a subfield of $K$ containing $P$ such that $K$ is separable over $h$. Then every regular subfield of $K$ containing $h$ is the field of constants of a set of higher 
derivations on $K$ if and only if (1) the transcendence degree of $K$ over $h$ is finite and (2) $K$ has a separating transcendency basis over $h$.

The Galois theory established in [4] required that $K$ be finitely generated over the distinguished regular subfields. In view of (4.2) we see that the correspondence can be extended to regular subfields $h$ such that $K$ has a finite separating transcendency basis over $h$. In part, the Galois correspondence can now be stated as follows.

(4.3) THEOREM. Assume $K$ has a finite separating transcendency basis over a regular subfield $h$ containing $P$. Then there exists a one-to-one correspondence between the regular subfields of $K$ containing $h$ and Galois subgroups of $H_{h}^{\infty}(K)$.

The characterization of the Galois subgroups remains the same as in [4]. Moreover, (4.2) shows the condition that $K$ have a finite separating transcendency basis over $h$ to be the most general we can impose and maintain a complete correspondence in that all regular subfields of $K$ containing $h$ will be fields of constants of sets of higher derivations.

\section{REFERENCES}

1. N. Heerema, Convergent higher derivations on local rings, Trans. Amer. Math. Soc. 132 (1968), 31-44. MR 36 \#6406.

2. - Derivations and embeddings of a field in its power series ring. II, Michigan Math. J. 8 (1961), 129-134. MR 25 \#69.

3. M. Weisfeld, Purely inseparable extensions and higher derivations, Trans. Amer. Math. Soc. 116 (1965), 435-449. MR 33 \#122.

4. N. Heerema and J. Deveney, Galois theory for fields $K / k$ finitely generated, Trans. Amer. Math. Soc. (to appear).

5. S. Mac Lane, Modular fields. I. Separating transcendency bases, Duke Math. J. 5 (1939), 372-393.

Department of Mathematics, Florida State University, Tallahassee, Florida 32306 